\title{
(C) OPEN ACCESS \\ Intrathecal baclofen therapy versus conventional medical management for severe poststroke spasticity: results from a multicentre, randomised, controlled, open-label trial (SISTERS)
}

\author{
Michael Creamer, ${ }_{1}^{1}$ Geoffrey Cloud ${ }^{2,3}$ Peter Kossmehl, ${ }^{4}$ Michael Yochelson, ${ }^{5,6}$ \\ Gerard E Francisco, ${ }^{7,8}$ Anthony B Ward ${ }^{9}$ Jörg Wissel, ${ }^{10}$ Mauro Zampolini, ${ }^{11}$ \\ Abdallah Abouihia, ${ }^{12}$ Nathalie Berthuy, ${ }^{12}$ Alessandra Calabrese, ${ }^{12}$ Meghann Loven, ${ }^{13}$ \\ Leopold Saltuari ${ }^{14,15}$
}

- Additional material is published online only. To view please visit the journal online (http://dx.doi.org/10.1136/ jnnp-2017-317021).

For numbered affiliations see end of article.

Correspondence to Dr Michael Creamer, Central Florida Pain Relief Centers, Orlando, Florida, USA: mcreamer@surgerypartners.com

Received 4 August 2017 Revised 13 November 2017 Accepted 5 December 2017 Published Online First 11 January 2018
Check for updates

To cite: Creamer M, Cloud G, Kossmehl P, et al. J Neurol Neurosurg Psychiatry 2018:89:642-650.

\begin{abstract}
Background Intrathecal baclofen (ITB) is a treatment option for patients with severe poststroke spasticity (PSS) who have not reached their therapy goal with other interventions.

Methods 'Spasticity In Stroke-Randomised Study' (SISTERS) was a randomised, controlled, open-label, multicentre phase IV study to evaluate the efficacy and safety of ITB therapy versus conventional medical management (CMM) with oral antispastic medications for treatment of PSS. Patients with chronic stroke with spasticity in $\geq 2$ extremities and an Ashworth Scale (AS) score $\geq 3$ in at least two affected muscle groups in the lower extremities (LE) were randomised (1:1) to ITB or CMM. Both treatment arms received physiotherapy throughout. The primary outcome was the change in the average $A S$ score in the LE of the affected body side from baseline to month 6 . Analyses were performed for all patients as randomised (primary analysis) and all randomised patients as treated (safety analysis).

Results of 60 patients randomised to ITB $(n=31)$ or CMM ( $n=29), 48$ patients (24 per arm) completed the study. The primary analysis showed a significant effect of ITB therapy over CMM (mean AS score reduction, -0.99 (ITB) vs -0.43 (CMM); Hodges-Lehmann estimate, $-0.667(95.1 \% \mathrm{Cl}-1.0000$ to -0.1667$) ; \mathrm{P}=0.0140)$. More patients reported adverse events while receiving ITB (24/25 patients, 96\%; 149 events) compared with CMM (22/35, 63\%; 77 events), although events were generally consistent with the known safety profile of ITB therapy.
\end{abstract}

Conclusions These data support the use of ITB therapy as an alternative to CMM for treatment of generalised PSS in adults.

Trial registration number NCT01032239; Results.

\section{INTRODUCTION}

Poststroke spasticity (PSS) is a common cause of disability in stroke survivors, reportedly occurring in $17 \%-43 \%$ of patients during the first year. ${ }^{1}$ Without effective treatment, the excessive muscle tone can cause pain and stiffness, deformity and reduced range of movement, significantly impairing the patient's quality of life. Management of severe PSS typically requires a multifaceted approach involving physical therapy and rehabilitation techniques and/or pharmacological treatments. ${ }^{23}$ Oral antispastics, particularly baclofen, have long been the cornerstone of treatment although they often lead to suboptimal results or cause adverse effects at therapeutic doses. ${ }^{45}$ While chemodenervation with botulinum neurotoxin type A has become the treatment of choice for focal spasticity, ${ }^{67}$ it may be inadequate for controlling symptoms over longer periods, particularly in severely affected patients with multifocal/segmental disabling spasticity.

Intrathecal baclofen (ITB) therapy is indicated for use in severe, chronic spasticity of cerebral or spinal origin, including generalised spastic hypertonia following stroke. Evidence for its effectiveness in treatment of spasticity mainly comes from controlled studies in cerebral palsy and multiple sclerosis, and no randomised trial to date has evaluated ITB therapy for PSS. In previous non-randomised studies in patients with stroke, ITB therapy was effective in reducing spastic tone in the extremities, increasing motor strength, reducing pain, improving gait and walking speed in ambulatory patients, as well as increasing functional independence and improving the patient's quality of life..$^{8-15}$ Despite this potential therapeutic value, $<1 \%$ of patients with stroke with severe spasticity are currently treated with ITB therapy. ${ }^{1}$

The 'Spasticity In Stroke-Randomised Study' (SISTERS) aimed to demonstrate the superior effect of ITB therapy over oral medication (conventional medical management (CMM)) on severe spasticity in patients with stroke after 6-month active treatment and to generate safety data for ITB therapy and CMM.

\section{METHODS}

Study design

SISTERS was a randomised, controlled, open-label, two-arm, parallel-group, phase IV study conducted in rehabilitation hospitals at 11 European centres (Austria, Belgium, Germany, Italy, the Netherlands, Spain, UK, Slovenia) and 7 US centres. The study is registered at ClinicalTrials.gov (number NCT01032239). 


\begin{tabular}{|c|c|c|}
\hline Characteristic & ITB $(n=31)$ & CMM $(n=29)$ \\
\hline \multicolumn{3}{|l|}{ Age, years } \\
\hline Mean (SD) & $56.1(11.1)$ & $55.7(8.6)$ \\
\hline Median (range) & $56.5(24.4,74.3)$ & $55.2(42.1,75.8)$ \\
\hline \multicolumn{3}{|l|}{ Race } \\
\hline White & $23(74.2 \%)$ & $23(79.3 \%)$ \\
\hline Other & $8(25.8 \%)$ & $6(20.7 \%)$ \\
\hline \multicolumn{3}{|l|}{ Sex } \\
\hline Male & $24(77.4 \%)$ & $18(62.1 \%)$ \\
\hline Female & $7(22.6 \%)$ & $11(37.9 \%)$ \\
\hline \multicolumn{3}{|l|}{ Type of stroke* } \\
\hline Cerebral ischaemic & $18(58.1 \%)$ & $12(41.4 \%)$ \\
\hline Cerebral haemorrhagic & $13(41.9 \%)$ & $17(58.6 \%)$ \\
\hline \multicolumn{3}{|l|}{ Time since last stroke, years } \\
\hline Mean (SD) & $4.95(3.56)$ & $4.55(3.73)$ \\
\hline Median (range) & $3.53(0.9,13.7)$ & $3.46(0.5,16.4)$ \\
\hline \multicolumn{3}{|l|}{ Spasticity duration, years } \\
\hline Mean (SD) & $4.53(3.79)$ & $3.91(3.01)$ \\
\hline Median (range) & $3.05(0.03,13.46)$ & $3.05(0.02,9.76)$ \\
\hline \multicolumn{3}{|l|}{ Ability to transfert } \\
\hline HLP & $22(71.0 \%)$ & $24(82.8 \%)$ \\
\hline LLP & $9(29.0 \%)$ & $5(17.2 \%)$ \\
\hline
\end{tabular}

${ }^{*}$ For the purposes of this study, a stroke was defined as any acute cerebrovascular accident (ischaemic or haemorrhagic).

tAbility to transfer was assessed by asking the patient to independently transfer from the wheelchair to bed without assistance. A patient who could transfer was classified as a HLP; otherwise he/she was classified as a LLP.

CMM, conventional medical management; HLP, high-level functional patient; ITB, intrathecal baclofen; ITT, intention-to-treat; LLP, low-level functional patient.

\section{Patients}

Potential participants were identified by the study team during clinic visits and by searching hospital patient databases. Eligible patients were men or women aged 18-75 years with a poststroke duration $>6$ months and generalised spasticity, who had not reached their therapy goal with other treatment interventions (eg, physiotherapy, botulinum toxin injection and oral medication). All patients had spasticity in at least two extremities and an Ashworth Scale (AS) score $\geq 3$ in a minimum of two muscle groups of the lower extremities (LE) on the affected body side. Exclusion criteria were: known baclofen sensitivity; uncontrolled refractory epilepsy; active systemic infection; presence of a cardiac pacemaker, implantable cardioverter defibrillator, implantable neurostimulator, or drug delivery device; use of oral vitamin $\mathrm{K}$ antagonists; use of botulinum toxin within the 4 months prior to inclusion; and inability/unwillingness of the patient/family to participate in long-term ITB therapy management. Chemodenervation (including botulinum toxin) and surgery affecting limb mobility were prohibited during the study. All patients (or legal guardian) provided written informed consent before study participation.

\section{Randomisation}

Using an interactive web-based randomisation system, we assigned patients $(1: 1)$ to ITB therapy or CMM according to a computer-generated sequence prepared by Medtronic. Randomisation was stratified by 'clusters of centres' via the block permutation method employing randomly selected blocks of sizes 2 or 4 . Clusters were established by grouping centres with similar patient type (low-level or high-level functional patients
(LLP or HLP); see table 1 for definition) and country standards of care.

Due to the nature of the treatments, the study was not blinded to the investigator or patient. To avoid AS score assessment bias and inter-rater variability, all AS scores (except for those during the ITB test) were performed by the same blinded assessor.

\section{Procedures}

The study comprised a run-in phase (21 days for CMM arm and 2-25 days for ITB arm) and a 6-month active trial. Both treatment arms received physiotherapy throughout the study per a protocol that was predefined by the investigator at each centre before study start. Active phase follow-up visits were at week 6 (ITB arm only) and at months 3 and 6. Unscheduled visits could be made in both treatment arms (eg, for titration/refill for patients on ITB).

Lioresal Intrathecal (baclofen injection; Novartis (Europe)/ Saol Therapeutics (US)) was used for ITB therapy. ITB arm patients underwent an ITB trial between days 1 and 10 during the run-in phase to evaluate drug response. Patients could continue their oral antispastic medications during this phase. At the ITB test visit, the AS score was measured prior to and at several points during ITB therapy administration. Patients fulfilling the test success criterion (1-point drop in the AS score in three muscle groups in the affected LE) were implanted between days 2 and 25 with the marketed SynchroMed II infusion system (Medtronic). After implant, patients underwent a 6 -week titration period during which the ITB dose was increased until the desired clinical effect was achieved or reduced for side-effect management; oral antispastics were gradually reduced with complete discontinuation by week 6. Patients randomised to ITB who were not implanted remained on oral antispastic medications and physiotherapy until the study end.

CMM arm patients received a combination of oral antispastic medication (at least one of oral baclofen, tizanidine, diazepam (or other benzodiazepines), or dantrolene) and physiotherapy throughout the study. Oral antispastic medications were prescribed by the investigator at randomisation; medications (type and dose) were then reassessed at the end of the run-in phase at the second assessment visit (day 21 2 2), and could be adjusted as deemed necessary by the investigator at any time during the trial, in accordance with usual clinical practice and the needs of the individual patient.

The change in spastic hypertonia and muscle tone was measured via AS scores for six muscle groups in the LE (hip flexors, hip adductors, knee extensors, knee flexors, plantar flexors and ankle-dorsal flexors) and five in the upper extremities (UE: wrist flexors, elbow flexors, elbow extensors, shoulder abductors and shoulder adductors). The AS is a 5-point scale (1 (normal) to 5 (rigid)) that measures resistance to passive movement across a joint. ${ }^{16}$ Average AS score in the LE (or UE) was calculated as the average of AS scores on muscles of the LE (or UE) on the affected body side, defined as the side with the highest average AS score at baseline. Functional change was evaluated using the Functional Independence Measure (FIM) ${ }^{17}$; this assesses 18 items categorised into six domains (self-care, sphincter control, transfers, locomotion, communication and social cognition) which are grouped into motor and cognitive subscales. AS scores were measured at the baseline, second assessment (CMM arm), ITB test (ITB arm), week 6 (ITB arm), month 3 and month 6 visits. The FIM was performed at baseline, month 3 and month 6 . 


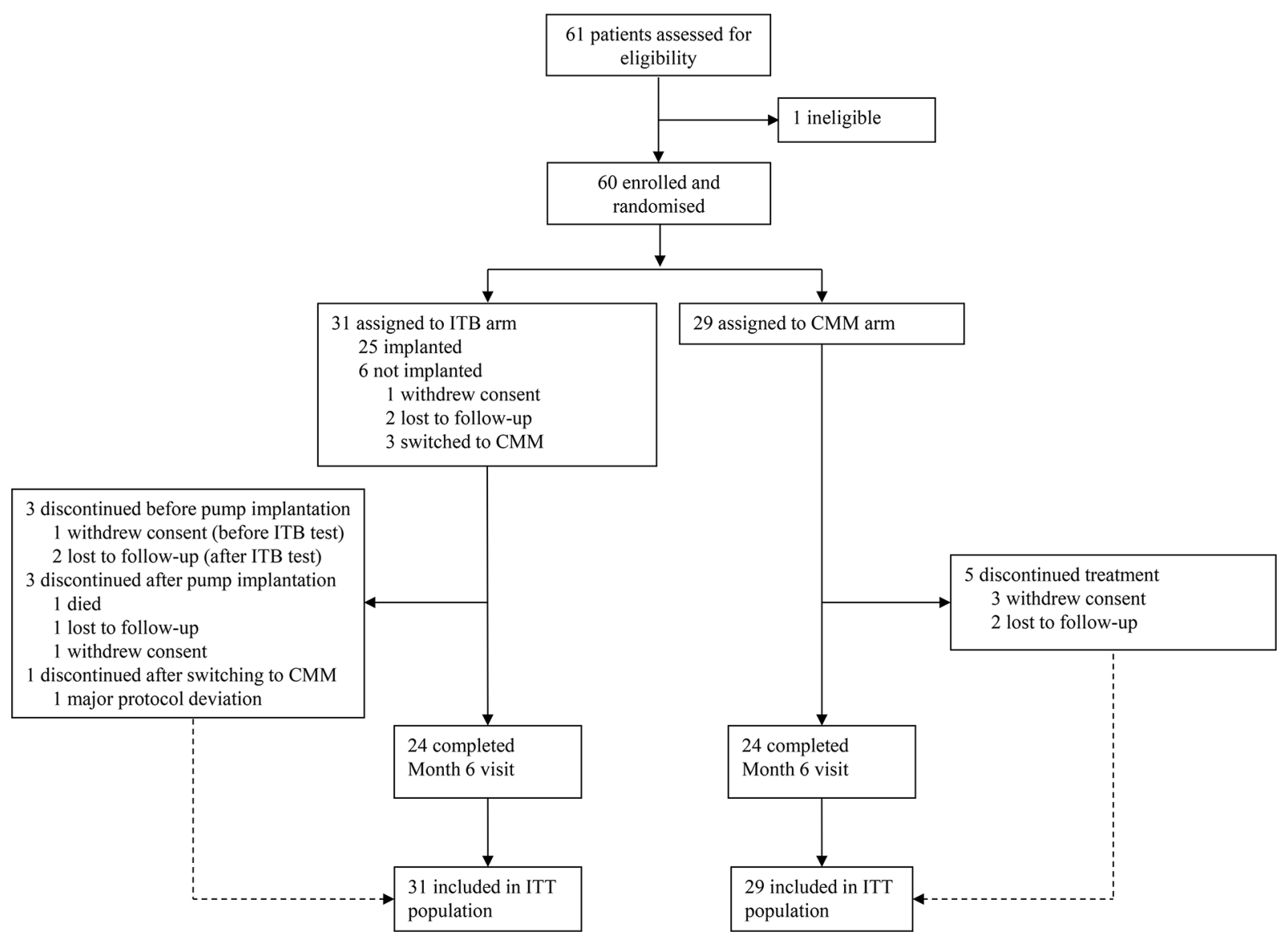

Figure 1 CONSORT (Consolidated Standards of Reporting Trials) flow diagram. CMM, conventional medical management; ITB, intrathecal baclofen; ITT, intention-to-treat.

Safety was primarily assessed by recording adverse events (AEs) throughout the study. The investigator classified AEs according to seriousness, severity and relationship to study drug (Lioresal Intrathecal in the ITB arm and oral antispastic medication in the CMM arm; adverse drug reactions (ADRs)) and/or device (ITB arm only; adverse device effects (ADEs)). Procedure-related AEs were those with aetiology of surgery or anaesthesia. All ADRs and serious adverse device effects (SADEs) were assessed for expectedness versus the product labelling for Lioresal Intrathecal and SynchroMed II pump, respectively. Any unexpected serious ADR was classified as a suspected unexpected serious adverse reaction (SUSAR). All AEs were coded using the MedDRA dictionary V.13.0.

\section{Outcomes}

The primary outcome was the change in spastic hypertonia and muscle tone in the affected lower limb, as measured by the change in average AS score in the LE of the affected body side from baseline to month 6. Key secondary outcomes (see the online supplementary appendix table S1 for full list) included assessment of safety (primarily AEs), muscle tone in the UE of the affected side and function assessed by the FIM (total score, motor and cognition sub scores and individual FIM dimensions).

\section{Statistics}

We calculated that 66 patients (33 per treatment arm) were required to test the null hypothesis (no difference in the change of the average AS score from baseline to month 6 between ITB and CMM) with $90 \%$ power. Allowing for $25 \%$ dropout in both arms, the study initially planned to randomise 88 patients (44 per arm). Due to patient recruitment difficulties, however, the sample size calculation was reassessed during the study. With a study power reduction (to 80\%) and lower than expected observed dropout (20\%), we calculated that 60 randomised patients (30 per arm) were required.

A blinded interim analysis was performed to validate the assumptions regarding the initial sample size assessment and to evaluate efficacy and study futility. To preserve the overall error risk of $\alpha=0.05$, an error risk of $\alpha 1=0.001$ was reserved for the interim analysis. Following the interim results, which demonstrated that there was no need to reassess the sample size and that futility could not be claimed, the data safety monitoring board recommended that the trial be continued without modification.

The intention-to-treat population (ITT; all patients as randomised) was the primary analysis set for all planned efficacy analyses, and the per protocol (PP) population (patients from the ITT who did not have any major protocol deviations and did not exit the study prematurely) was used for sensitivity analyses. A modified-ITT (mITT) population was also used for sensitivity analysis of the primary efficacy variable to show 'best-case difference' between groups; this consisted of all randomised patients analysed as treated, that is patients who were ITB-implanted (ITB-I group) versus patients treated with CMM plus patients randomised to the ITB arm who were not implanted (CMM+ non implanted group). An additional set (ITT completers; all patients in the ITT population who completed a 6-month follow-up) was used for an ad-hoc sensitivity analysis 


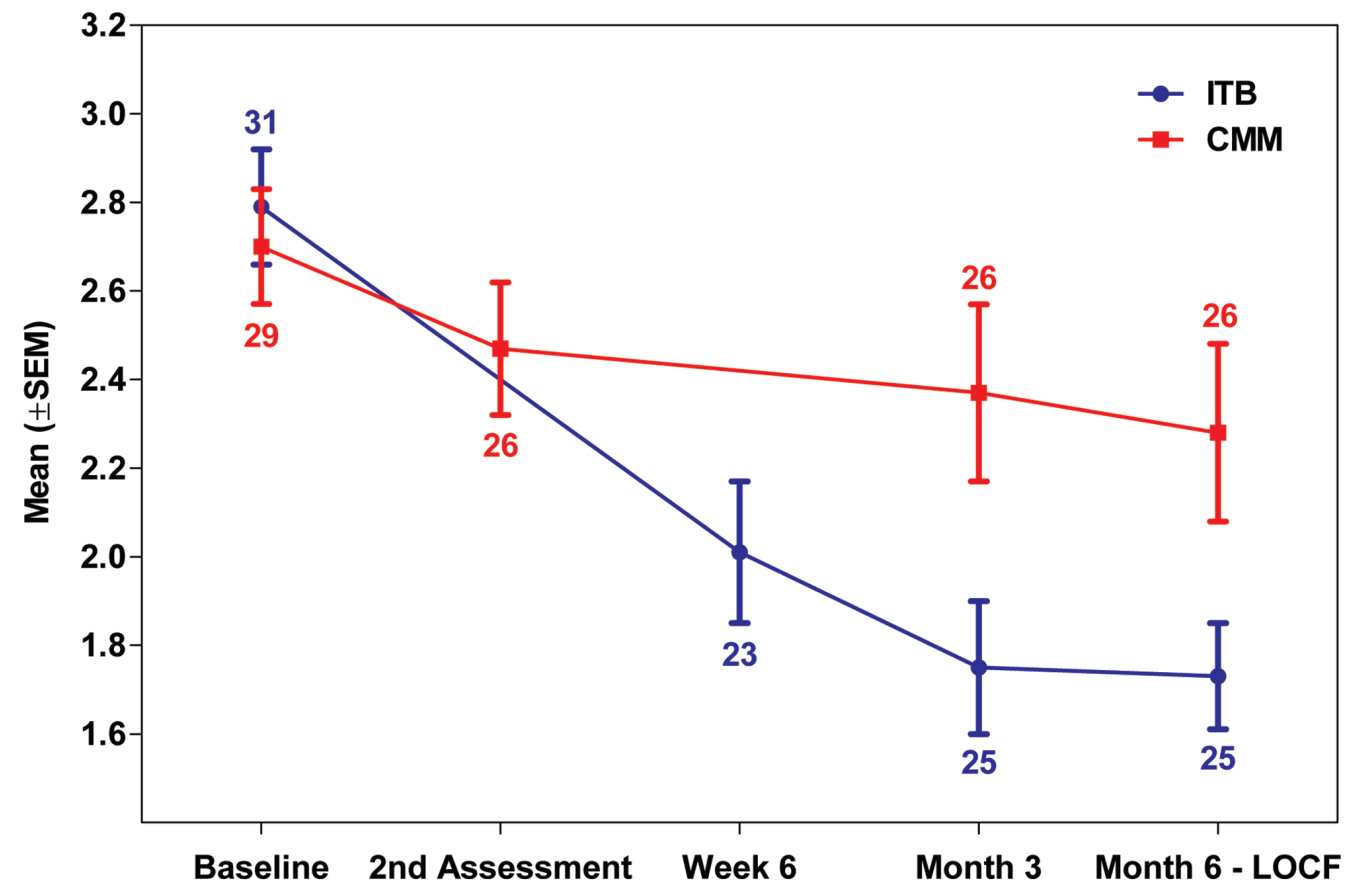

B

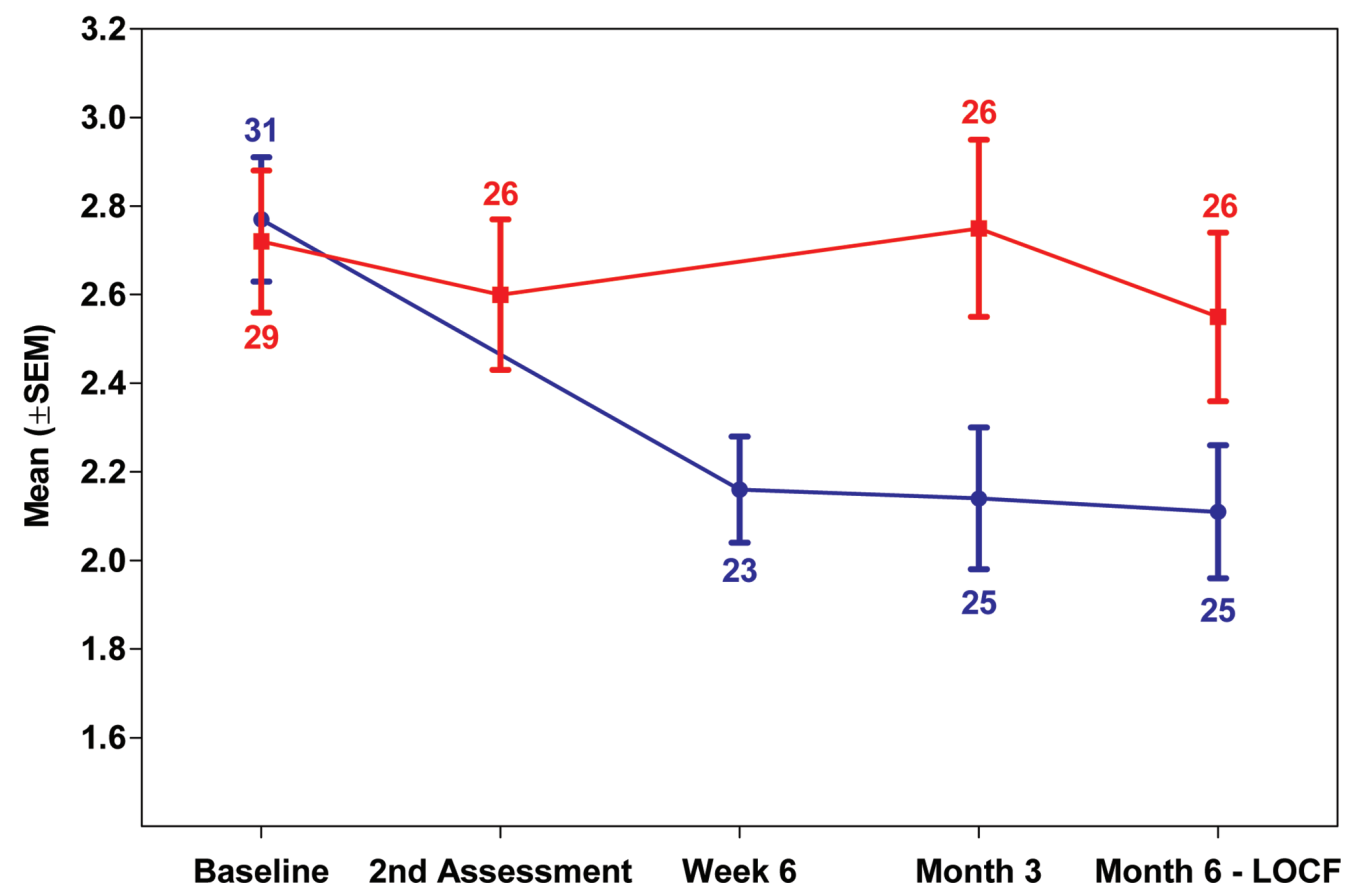

Figure 2 Average AS score for the lower and upper limbs by study visit (ITT population). Mean (SEM) values for average AS score in the LE (panel A) and UE (panel B) of the affected side across patients by treatment arm (ITB vs CMM) and study visit. LOCF imputation was performed for the month 6 assessment using month 3 data; the number of patients analysed per visit is indicated. The second assessment (CMM arm) was done on day 21. AS, Ashworth Scale; CMM, conventional medical management (arm); ITB, intrathecal baclofen (arm); ITT, intention-to-treat; LE, lower extremities; LOCF, last observation carried forward; UE, upper extremities.

of the primary outcome. Safety analyses were performed on the mITT for all AEs and study drug-related AEs, or ITT for device-related and procedure-related AEs (as non-implanted patients in the ITB arm could have experienced these events as a result of the ITB test).

The primary efficacy outcome was compared between ITB therapy and CMM using the Wilcoxon Rank Sum test and by calculating the Hodges-Lehmann estimator, as was the change in the average AS score in the UE (secondary outcome). Changes in the FIM score, motor and cognition subscores, and six FIM domains from baseline to month 6 were compared using the Wilcoxon test. Last observation carried forward (LOCF) imputation was used for analysis of primary and secondary variables in the ITT and mITT populations; patients with data missing for 
Table 2 Analysis of change from baseline to month 6 in the average AS score in the LE (primary outcome) and UE (secondary outcome)

\begin{tabular}{|c|c|c|c|c|c|}
\hline & & \multicolumn{2}{|c|}{ Change from baseline to month $6^{*}$} & \multirow[b]{2}{*}{$P$ valuet } & \multirow[b]{2}{*}{ Hodges-Lehmann estimate $\ddagger$} \\
\hline & & ITB $(n=31)$ & CMM $(n=29)$ & & \\
\hline \multicolumn{6}{|c|}{ Average AS score in the LE of the affected side } \\
\hline \multirow[t]{3}{*}{ ITT } & $\mathrm{N}$ & 25 & 26 & 0.0140 & $-0.667(95.1 \% \mathrm{Cl}-1.0000$ to -0.1667$)$ \\
\hline & Mean (SD) & $-0.99(0.75)$ & $-0.43(0.72)$ & & \\
\hline & Median (min, max) & $-0.83(-2.3,0.7)$ & $-0.25(-2.2,0.7)$ & & \\
\hline \multirow[t]{3}{*}{ Per protocol } & $\mathrm{N}$ & 15 & 20 & 0.0592 & $-0.667(95.1 \% \mathrm{Cl}-1.1667$ to 0.0000$)$ \\
\hline & Mean (SD) & $-1.09(0.63)$ & $-0.48(0.82)$ & & \\
\hline & Median (min, max) & $-0.83(-2.3,0.0)$ & $-0.42(-2.2,0.7)$ & & \\
\hline \multirow[t]{3}{*}{ Modified ITT } & $\mathrm{N}$ & 22 & 29 & 0.0019 & $-0.667(95.1 \% \mathrm{Cl}-1.1667$ to -0.3333$)$ \\
\hline & Mean (SD) & $-1.11(0.68)$ & $-0.39(0.71)$ & & \\
\hline & Median (min, max) & $-0.92(-2.3,0.3)$ & $-0.33(-2.2,0.7)$ & & \\
\hline \multirow{3}{*}{$\begin{array}{l}\text { ITT } \\
\text { completers }\end{array}$} & $\mathrm{N}$ & 24 & 24 & 0.0240 & $-0.667(95.1 \% \mathrm{Cl}-1.0000$ to -0.1667$)$ \\
\hline & Mean (SD) & $-1.01(0.75)$ & $-0.47(0.73)$ & & \\
\hline & Median (min, max) & $-0.83(-2.3,0.7)$ & $-0.33(-2.2,0.7)$ & & \\
\hline \multicolumn{6}{|c|}{ Average AS score in the UE of the affected side } \\
\hline \multirow[t]{3}{*}{ ITT } & $\mathrm{N}$ & 25 & 26 & 0.0042 & $-0.600(95 \% \mathrm{Cl}-1.0000$ to -0.2000$)$ \\
\hline & Mean (SD) & $-0.66(0.59)$ & $-0.17(0.70)$ & & \\
\hline & Median (min, max) & $-0.60(-1.6,0.6)$ & $0.00(-2.4,0.6)$ & & \\
\hline \multirow[t]{3}{*}{ Per protocol } & $\mathrm{N}$ & 15 & 20 & 0.0494 & $-0.600(95 \% \mathrm{Cl}-1.0000$ to 0.0000$)$ \\
\hline & Mean (SD) & $-0.64(0.58)$ & $-0.22(0.78)$ & & \\
\hline & Median (min, max) & $-0.60(-1.4,0.6)$ & $0.00(-2.4,0.6)$ & & \\
\hline
\end{tabular}

\footnotetext{
${ }^{*}$ For the ITT and modified-ITT populations, the calculation was based on LOCF imputation using data for month 3 assessment in patients for whom month 6 data were not available. Patients with data missing for both months 3 and 6 were excluded from the analysis.

tWilcoxon Rank Sum test: comparison of the difference in the change of AS score from baseline to month 6 between groups.

¥Hodges-Lehmann estimate of the location shift (asymptotic method) between groups with $95.1 \% \mathrm{Cl}$ for average AS score in the LE and $95 \% \mathrm{Cl}$ for average AS score in the UE. AS, Ashworth Scale; CMM, conventional medical management; ITB, intrathecal baclofen; ITT, intention-to-treat; LE, lower extremities; LOCF, last observation carried forward; UE, upper extremities.
}

both months 3 and 6 were excluded from the analysis. Statistical tests were performed at the two-sided alpha level of $4.9 \%$ (primary efficacy variable) or 5\% (secondary/additional variables), with no adjustment of type 1 error due to multiplicity. All analyses were done using SAS software V.9.4.

\section{RESULTS}

Between 16 December 2009 and 31 December 2015, 60 patients were enrolled and randomised to receive ITB therapy (31) or CMM (29) and comprised the ITT population (figure 1). Thirty of 31 patients in the ITB arm attended an ITB test visit (one withdrew beforehand), 29 underwent an ITB test and 25 were implanted with the device. Of the five patients who attended an
ITB test visit and were not implanted, two met the test criterion but were lost to follow-up, one patient was not tested (the patient was deemed unsuitable for surgery and switched to CMM), and two patients failed the ITB test and were also switched to CMM. All six patients randomised to ITB who were not implanted were grouped with patients on CMM ('CMM+non-implanted group') for analysis in the mITT population, where appropriate. In total, 48 patients in the ITT population ( 24 in each arm) completed the month 6 visit (ITT completers), including 22 patients with ITB-I. Twelve patients prematurely terminated the study (7/31 $(23 \%)$ and 5/29 (17\%) in the ITB and CMM arms, respectively). The PP population comprised 15 patients with ITB-I and 20 patients on CMM (CMM+non-implanted).

Table 3 FIM total score and FIM motor and cognition subscores (ITT population)

\begin{tabular}{|c|c|c|c|c|c|c|c|}
\hline & & \multicolumn{2}{|l|}{ FIM total } & \multicolumn{2}{|c|}{ FIM motor subscore } & \multicolumn{2}{|c|}{ FIM cognition subscore } \\
\hline & & ITB & CMM & ITB & CMM & ITB & CMM \\
\hline \multirow[t]{3}{*}{ Baseline } & $\mathrm{N}$ & 31 & 29 & 31 & 29 & 31 & 29 \\
\hline & Mean (SD) & $89.23(28.76)$ & $96.10(19.45)$ & $59.77(24.96)$ & $66.45(14.96)$ & $29.45(7.66)$ & $29.66(7.41)$ \\
\hline & $\begin{array}{l}\text { Median } \\
(\min , \max )\end{array}$ & $101.00(25.0,126.0)$ & $103.00(47.0,124.0)$ & $72.00(13.0,91.0)$ & $71.00(41.0,89.0)$ & $34.00(11.0,35.0)$ & $33.00(6.0,35.0)$ \\
\hline \multirow{3}{*}{$\begin{array}{l}\text { Change from baseline } \\
\text { to month } 6^{*}\end{array}$} & $\mathrm{~N}$ & 25 & 26 & 25 & 26 & 25 & 26 \\
\hline & Mean (SD) & $2.68(10.31)$ & $-2.58(11.00)$ & $2.20(8.90)$ & $-0.96(8.14)$ & $0.48(3.92)$ & $-1.62(4.92)$ \\
\hline & $\begin{array}{l}\text { Median } \\
(\min , \max )\end{array}$ & $0.00(-21.0,27.0)$ & $-2.00(-26.0,33.0)$ & $0.00(-21.0,22.0)$ & $-3.00(-18.0,26.0)$ & $0.00(-6.0,15.0)$ & $0.00(-15.0,8.0)$ \\
\hline$P$ valuet & & 0.0540 & & 0.0964 & & 0.2569 & \\
\hline
\end{tabular}

${ }^{*}$ Calculation was based on LOCF imputation using data for month 3 assessment in patients for whom month 6 data were not available. Patients with data missing for both months 3 and 6 were excluded from the analysis.

tComparison of the change from baseline to month 6 between groups using the Wilcoxon test.

CMM, conventional medical management; FIM, Functional Independence Measure; ITB, intrathecal baclofen; ITT, intention-to-treat; LOCF, last observation carried forward. 


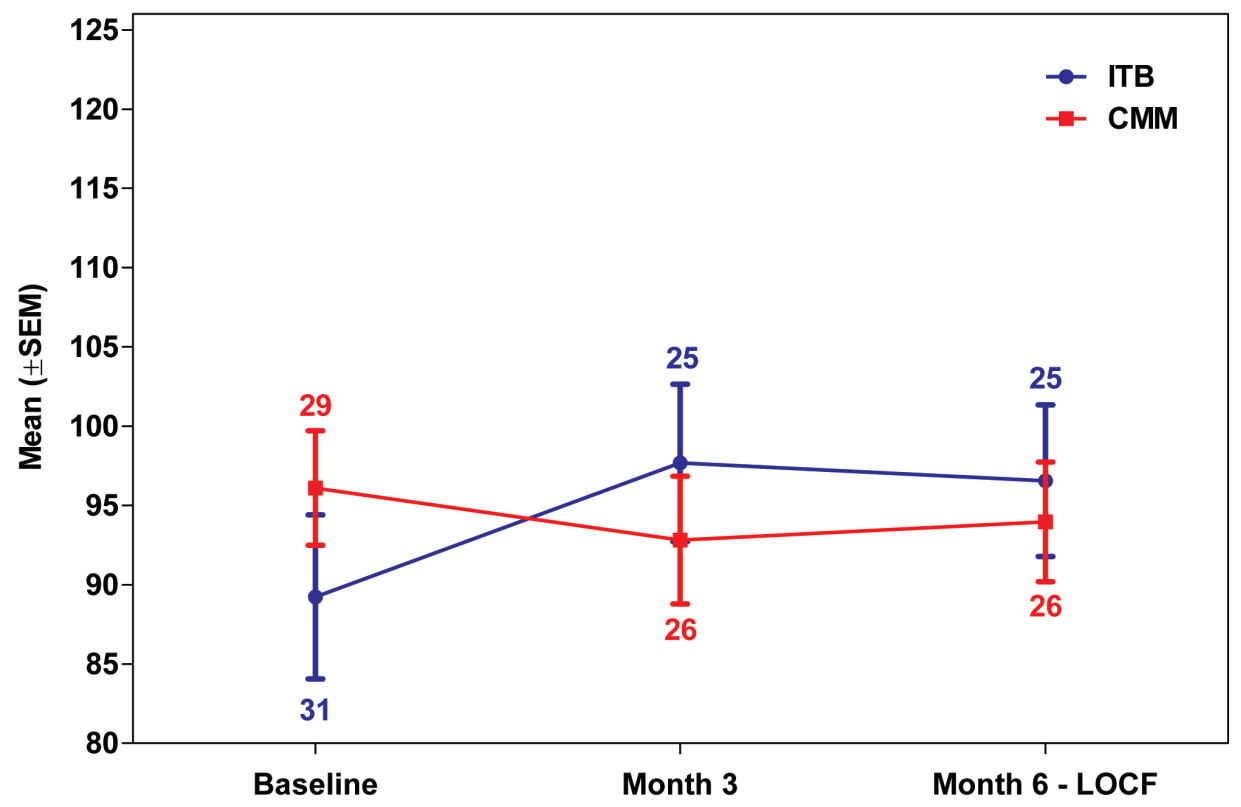

Figure 3 FIM total score by study visit (ITT population). LOCF imputation was performed for the month 6 assessment using month 3 data; the number of patients analysed per visit is indicated. CMM, conventional medical management (arm); FIM, Functional Independence Measure ; ITB, intrathecal baclofen (arm); ITT, intention-to-treat; LOCF, last observation carried forward.

Baseline demographic and clinical characteristics were generally comparable between the two treatment arms (table 1), although the ITB arm had a greater proportion of LLP and fewer patients with haemorrhagic stroke. In the ITB arm, the mean (SD) daily ITB dose $(\mu \mathrm{g})$ increased from 79.0 (38.1) at implant to 247.7 (163.1) at week 6 (end of titration); a further increase was observed during the follow-up period (279.3 (218.7) at month 3 ; 296.6 (246.5) at month 6). In the CMM arm, the most common oral antispastic medications taken during the randomised period (see the online supplementary appendix 2) were oral baclofen (23/29 patients (79\%); mean daily dose, $37.2(26.0) \mathrm{mg})$ and tizanidine (6/29 (21\%); 6.3 (3.3) $\mathrm{mg})$.

Both treatment arms were comparable regarding the total number of physiotherapy sessions per week (2.6 (1.3) and 2.8 (1.2) for ITB and CMM arms, respectively) and duration per session (56 (16) and 52 (11) mins, respectively). The mean number of all study visits (including unscheduled visits) was 14.1 (8.7) in the ITB arm and 3.8 (1.1) in the CMM arm.

\section{Spastic hypertonia and muscle tone}

There was an overall decrease (improvement) in average AS score in the LE from baseline to month 6 in both treatment arms (figure 2A), with a greater improvement observed in the ITB arm (mean change, $-0.99(0.75)$ for ITB and $-0.43(0.72)$ for $\mathrm{CMM}$ ). The primary analysis showed a significant effect in favour of ITB therapy over CMM in the ITT population $(\mathrm{P}=0.0140$; HL estimate of $-0.667(95.1 \% \mathrm{CI}-1.0000$ to -0.1667$))$. The robustness of the treatment effect was confirmed with sensitivity analyses (table 2), which showed the same effect magnitude. A significant effect of ITB therapy over CMM was observed for mITT $(\mathrm{P}=0.0019)$ and ITT completers $(\mathrm{P}=0.0240$; ad-hoc analysis) populations, and a trend towards improvement for ITB therapy was observed in the PP population $(\mathrm{P}=0.0592)$.

For spasticity in the UE of the affected side (table 2 and figure 2B), a significant effect of ITB therapy over CMM was observed for the change in the average AS score from baseline to month 6, both for the ITT (HL estimate -0.600 (95\% CI -1.0000 to -0.2000$) ; \mathrm{P}=0.0042)$ and $\mathrm{PP}(-0.600(95 \% \mathrm{CI}$ -1.0000 to 0.0000$) ; \mathrm{P}=0.0494$ ) populations.

\section{Functional independence}

An improvement in FIM total score from baseline to month 6 occurred in the ITB arm $(+2.68(10.31))$, while a worsening occurred in the CMM arm (-2.58 (11.00)) (table 3 and figure 3); the analysis showed a trend towards a treatment effect in favour of ITB over CMM $(\mathrm{P}=0.0540)$. Trends towards an improvement from baseline were also noted for the motor subscore $(\mathrm{P}=0.0964)$ and self-care score $(\mathrm{P}=0.0964)$ (table 3 and online supplementary appendix S3). There were no significant differences between the two treatments with respect to changes in the cognition subscore or for other individual FIM domains.

\section{Safety}

Treatment-emergent AEs occurred more frequently in the ITB-I group (24/25 (96\%) patients, 149 events) than in the CMM+non implanted group (22/35 (63\%) patients, 77 events) (table 4$)$, although the vast majority in both groups were transient and mild/moderate in intensity. In the ITB-I group, approximately half of all events (69 of 149) occurred during the titration period.

Fifty-eight treatment-emergent serious adverse events (SAEs) occurred: 34 events for $12 / 25$ (48\%) patients in the ITB-I group and 24 for 10/35 (29\%) patients in the CMM+non-implanted group. No individual SAE was reported by more than two patients in either group (online supplementary appendix S4). One patient with ITB-I died after week 6 due to accidental alcohol poisoning which was not considered by the investigator to be directly related to the study drug or device. Eight events were classified as SADRs (probably/possibly related to study drug), including seven events in 6/25 (24\%) patients in the ITB-I group (two events of urinary retention, and one each 
Table 4 Summary of AEs and SAEs with ITB therapy or CMM (modified ITT population)

\begin{tabular}{lll}
\hline & $\begin{array}{l}\text { ITB-I } \\
(\mathbf{n}=25)\end{array}$ & $\begin{array}{l}\text { CMM+non- } \\
\text { implanted } \\
(\mathbf{n}=35)\end{array}$ \\
\hline All AEs & $24(96.0)(155)$ & $22(62.9)(79)$ \\
\hline All serious AEs & $13(52.0)(35)$ & $10(28.6)(24)$ \\
\hline Resulted in patient death & $1(4.0)(1)^{*}$ & $0(0.0)(0)$ \\
\hline All treatment-emergent AEst & $24(96.0)(149)$ & $22(62.9)(77)$ \\
\hline Drug-related (ADRs) & $14(56.0)(39) \ddagger$ & $6(17.1)(12)$ \\
\hline Device-related (ADEs) & $9(36.0)(16)$ & NA \\
\hline Procedure-related & $6(24.0)(9)$ & $1(2.9)(1) \S$ \\
\hline Serious treatment-emergent AEs & $12(48.0)(34)$ & $10(28.6)(24)$ \\
\hline Drug-related (SADRs) & $6(24.0)(7)$ & $1(2.9)(1)$ \\
\hline Device-related (SADEs) & $4(16.0)(4) \uparrow$ & NA \\
\hline Procedure-related & $1(4.0)(1)^{* *}$ & $0(0.0)(0)$ \\
\hline Treatment-emergent AEs by & & \\
intensity & & $19(54.3)(43)$ \\
\hline Mild & $22(88.0)(89)$ & $13(37.1)(20)$ \\
\hline Moderate & $16(64.0)(33)$ & $4(11.4)(4)$ \\
\hline Severe & $7(28.0)(10)$ & \\
\hline
\end{tabular}

Data presented are number of patients (\% of patients) and (number of AE reports).

*Event of accidental alcohol poisoning.

TAEs were treatment-emergent if not present prior to study treatment initiation or if any event already present worsened in either intensity or frequency following exposure to study treatment. Start of treatment refers to the start of the ITB test for patients in the ITB arm and randomisation for patients in the CMM arm.

¥Includes two unexpected events of faecal impaction reported for two patients with ITB-I (one event was classified as a SUSAR).

$\S$ One patient randomised to the ITB arm experienced a procedure-related event during the ITB test but was not implanted.

ITTe four SADEs included device dislocation, device occlusion, implant site infection, and intracranial hypotension.

** One event of pain was considered related to the implant procedure. $A D E$, adverse device effect; $A D R$, adverse drug reaction; $A E$, adverse event; CMM, conventional medical management; ITB-I, intrathecal baclofen-implanted; ITT, intention-to-treat; SADE, serious adverse device effect; SADR, serious adverse drug reaction; SAE, serious adverse event; SUSAR, suspected unexpected serious adverse reaction. of constipation, faecal impaction, epileptic seizure, peripheral oedema and hypotension), and one case of epileptic seizures in a patient on CMM. The faecal impaction event was unexpected based on the Lioresal Intrathecal product label and therefore classified as a SUSAR. Four events in four separate patients with ITB-I were classified as SADEs (device dislocation, device occlusion, implant site infection and intracranial hypotension), of which three led to device revision. One SAE (severe subscapular pain) in a patient with ITB-I was considered related to the implant procedure. Apart from the patient who died, no other patient discontinued the study due to an AE, and no AEs led to discontinuation of ITB therapy after implantation.

The most common ADR in the ITB-I group was muscular weakness, reported with a higher frequency than in the $\mathrm{CMM}+$ non-implanted group $(4 / 25(16 \%)$ vs $1 / 35(3 \%)$ patients, respectively); this was followed by fall, hypotonia and urinary retention (each reported for 3/25 (12\%) patients in the ITB-I group only). Somnolence was the only ADR to be reported for more than one patient in the $\mathrm{CMM}+$ non-implanted group $(3 / 35$ (9\%) patients vs none in the ITB-I group). Two events were classified as unexpected; both ADRs of faecal impaction (including one SUSAR) were reported in two patients with ITB-I. The most frequently reported ADEs were muscular weakness (three events in two patients with ITB-I), fall and hemiparesis (two events each in two patients with ITB-I). Six of these seven ADEs were also classified as ADRs. Ten procedure-related AEs were reported for $7 / 31$ (19\%) patients randomised to the ITB arm, of which 9 occurred in 6 patients with ITB-I, the most common events being pain and implant site pain ( 2 events each in 2 patients). In the ITB-I group, almost half of all treatment-related events (25/55 (45\%)) occurred between implant and week 6 (figure 4).

\section{DISCUSSION}

We observed a significant effect of treatment with ITB therapy over $\mathrm{CMM}$ for reduction of spastic hypertonia and muscle tone in the lower limb of the affected side over a 6-month follow-up. Importantly, the minimal clinically important improvement of 1 point

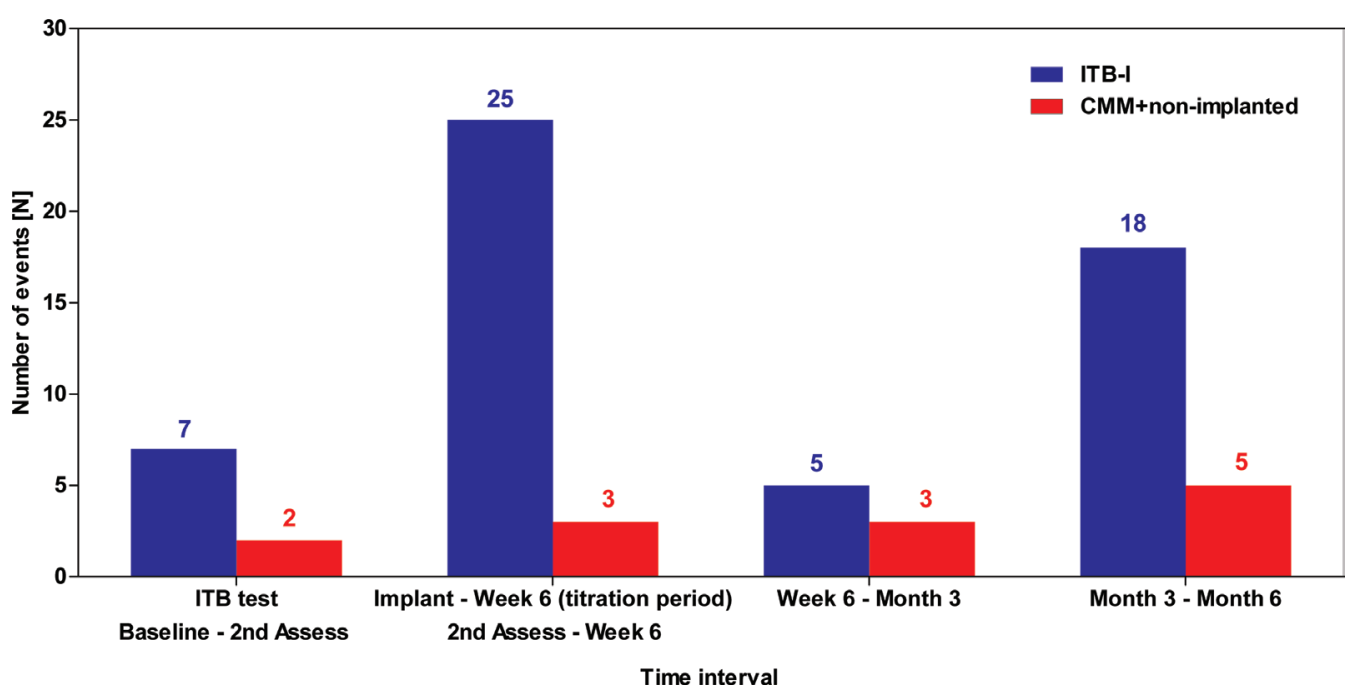

Figure 4 Frequency of treatment-related AEs (drug, device, procedure) over time during the study (modified ITT population). Second assessment: day $21 \pm 2$ (CMM arm); week 6: day 44-67 (ITB arm) and corresponds to day 67 for CMM. AE, adverse event; CMM, conventional medical management; ITB, intrathecal baclofen; ITB-I, ITB-implanted; ITT, intention-to-treat. 
for the average AS score ${ }^{18}$ (calculated over six muscle groups) was achieved in the ITB arm. The robustness of the treatment effect was confirmed by sensitivity analyses which all showed the same magnitude of effect, although the between-group difference did not reach statistical significance in the PP population, likely due to the reduced sample size. The observed difference occurred independently of any effect of physiotherapy as both treatment arms received a similar amount of physiotherapy. Although patients in the CMM arm were not managed according to a centralised treatment algorithm, some degree of improvement over baseline was observed, indicating active management of these patients during the study.

The observed spasticity reduction in the LE with ITB therapy confirms data from previous studies showing a reduction of muscle tone in the lower limb in ITB-treated patients with stroke. ${ }^{9} 101215$ We also observed a significant treatment effect for ITB over CMM for spasticity reduction in the upper limb, despite the fact that the study was not powered to detect treatment differences in secondary outcomes. This result agrees with two previous studies that showed a decrease from baseline in muscle tone in the upper limb with ITB therapy. 912

For the FIM total score, a positive trend for improvement with ITB therapy versus CMM was observed, despite the relatively short follow-up period. A comparable mean increase from baseline was reported for patients on ITB in the Ivanhoe et al' study at 12 months (+2.86 (SD 10.13)). ${ }^{12}$ While FIM motor and cognition subscores in our study tended to improve in the ITB arm and worsen in the CMM arm over 6 months, between-group differences were not statistically significant.

A higher overall rate of AEs occurred in the ITB-I group compared with the CMM+non-implanted group, although given the nature of the intervention this is not unexpected: patients with ITB-I underwent a surgical procedure followed by active titration of ITB therapy (with optimisation by week 6) with weaning of concomitant oral medication. Furthermore, given the higher average number of visits per patient in the ITB versus the CMM arm (14 and 4, respectively), this finding could be partially related to the increased opportunity for AE reporting in patients on ITB. Moreover, almost half of all treatment-related AEs for patients with implants occurred during the titration period when the ITB dose is adjusted to optimise therapy (typically increased until the first $\mathrm{AE}$ is observed). Observed treatment-related AEs in the ITB arm were in line with expected events based on the known safety profile of ITB therapy in this patient population, although two ADRs of faecal impaction in two separate patients were assessed as unexpected (one was a SUSAR). The four SADEs were all considered expected according to the device label.

The strengths of this study include its randomised, controlled design and that this is, to our knowledge, the first study to evaluate the efficacy of ITB therapy versus CMM for PSS management. Limitations include a lower than anticipated sample size owing to recruitment difficulties and that the study was not powered to detect treatment differences for secondary outcomes. In addition, the 6-month follow-up period was relatively short, particularly for evaluation of functional changes. Future studies with larger sample sizes and longer follow-up could allow identification of patient subgroups that will benefit most from ITB therapy.

In conclusion, our results suggest that intrathecal delivery of baclofen provides an improved therapeutic effect versus conventional oral medications, with a reduction of the AS score in both upper and lower limbs when used in conjunction with physiotherapy. The continuous infusion also confers prolonged and stable tone control. Overall, these data confirm and expand on existing literature and support the use of ITB therapy for treatment of generalised spasticity in adults with PSS.

\section{Author affiliations}

${ }^{1}$ Central Florida Pain Relief Centers, Orlando, Florida, USA

${ }^{2}$ St. George's University Hospitals NHS Foundation Trust, London, UK

${ }^{3}$ Department of Neurology, Alfred Health, Melbourne, Victoria, Australia

${ }^{4}$ Kliniken Beelitz GmbH Neurologische Rehabilitationsklinik, Beelitz-Heilstätten,

Germany

${ }^{5}$ MedStar National Rehabilitation Hospital, Washington DC, USA

${ }^{6}$ Shepherd Center, Atlanta, Georgia, USA

${ }^{7}$ Department of Physical Medicine and Rehabilitation, University of Texas Health

Science Center, Houston, Texas, USA

${ }^{8}$ TIRR Memorial Hermann Hospital, Houston, Texas, USA

${ }^{9}$ North Staffordshire Rehabilitation Centre, Haywood Hospital, Stoke on Trent, UK

${ }^{10}$ Department of Neurology, Neurological Rehabilitation and Physical Therapy,

Vivantes Hospital Spandau, Berlin, Germany

${ }^{11}$ Department of Rehabilitation, Ospedale di Foligno, USL Umbria 2, Perugia, Italy

${ }^{12}$ Neuromodulation Clinical, Medtronic International Trading Sarl, Tolochenaz,

Switzerland

${ }^{13}$ Medtronic Inc, Minneapolis, Minnesota, USA

${ }^{14}$ Abteilung für Neurologie, Landeskrankenhaus Hochzirl, Zirl, Austria

${ }^{15}$ Research Unit for Neurorehabilitation, South Tyrol, Bolzano, Italy

Correction notice Since this paper was first published online figure 1 has been updated.

Acknowledgements The authors would like to thank all participants and carers who participated in this study. They gratefully acknowledge the support and work of the study research team including the following principal/co-investigators: $\operatorname{Dr} R$ Van der Looven and Dr K Bouche (UZ Gent, Gent), Dr B Rubin (Design Neuroscience Center, Doral, FL), Dr S Khurana (University of Miami, Miami, FL), Dr S Moraleda (Hospital Universitario La Paz (Madrid), Dr A Bender (Therapiezentrum Burgau, Burgau), Dr J Ruijgrok (AZ Maastricht, Maastricht), Dr J Shilt (Saint Alphonsus Regional Medical Center, Boise, ID), Prof T Lejeune (UCL Saint-Luc, Brussels), Dr M Ebke (Rhein-Sieg-Klinik Dr Becker Klinikgesellschaft, Nuembrecht), Dr F Molteni (Villa Beretta, Costamasnaga, Lecco), Dr M Lawson (Tallahassee Neurological Clinic, Tallahassee, FL), Dr K Grabljevec (Univerzitetni Rehabilitacijski Institut Soca, Ljubljana), Dr Catherine Dalton (St George's Hospital, Tooting, London) and Dr H Matzak (Landeskrankenhaus Hochzirl, Zirl). They also acknowledge Dr Sarah Hopwood (Scinopsis, France) for medical writing assistance with this publication, paid by Medtronic

Contributors MC: contributed to acquisition of the data and drafted the manuscript for content. GC, PK, MY, GEF, JW and LP: contributed to acquisition of the data. GEF, ABW, JW and MZ: responsible for the study concept and interpretation of the data. LS: was responsible for study concept, coordination of the study and interpretation of the data. AA: designed the study with the Steering Committee and study manager and was the study statistician, providing advice and input on all statistical analyses. NB, ML and AC: responsible for management of the study and interpretation of the data. All authors: contributed to writing and editing of the manuscript and approved the final version.

Funding This work was supported by Medtronic International Trading Sàrl. MC, MZ and $L S$ report personal fees from Medtronic during the conduct of the study. GEF reports grants from Allergan, Ipsen, Merz and Mallinckrodt during the conduct of the study. JW reports personal fees from Medtronic during the conduct of the study, and personal fees from Allergan, Merz, Ipsen, and Medtronic outside the submitted work. $A A, N B, A C$ and $M L$ are all employees of Medtronic and report personal fees from Medtronic during the conduct of the study.

Disclaimer The funder (Medtronic International Trading Sàrl) had a role in the study design, as well as the collection, analysis and interpretation of data, and writing of the report.

Competing interests $M C, M Z$ and LS report personal fees from Medtronic during the conduct of the study. GF reports grants from Allergan, Ipsen, Merz and Mallinckrodt during the conduct of the study. JW reports personal fees from Medtronic during the conduct of the study, and personal fees from Allergan, Merz, Ipsen, and Medtronic outside the submitted work. AA, NB, AC and ML are all employees of Medtronic and report personal fees from Medtronic during the conduct of the study. All other authors declare no competing interests.

Ethics approval An IRB or EC and competent authorities (EU only) approved the study at each participating centre and country.

Provenance and peer review Not commissioned; externally peer reviewed.

Open Access This is an Open Access article distributed in accordance with the Creative Commons Attribution Non Commercial (CC BY-NC 4.0) license, which permits others to distribute, remix, adapt, build upon this work non-commercially, 
and license their derivative works on different terms, provided the original work is properly cited and the use is non-commercial. See: http://creativecommons.org/ licenses/by-nc/4.0/

(c) Article author(s) (or their employer(s) unless otherwise stated in the text of the article) 2018. All rights reserved. No commercial use is permitted unless otherwise expressly granted.

\section{REFERENCES}

1 Dvorak EM, Ketchum NC, McGuire JR. The underutilization of intrathecal baclofen in poststroke spasticity. Top Stroke Rehabil 2011;18:195-202.

2 Thibaut A, Chatelle C, Ziegler E, et al. Spasticity after stroke: physiology, assessment and treatment. Brain Inj 2013;27:1093-105.

3 Bakheit AM. The pharmacological management of post-stroke muscle spasticity. Drugs Aging 2012;29:941-7.

4 Ertzgaard P, Campo C, Calabrese A. Efficacy and safety of oral baclofen in the management of spasticity: a rationale for intrathecal baclofen. J Rehabil Med 2017:49:193-203.

5 Lindsay C, Kouzouna A, Simcox C, et al. Pharmacological interventions other than botulinum toxin for spasticity after stroke. Cochrane Database Syst Rev 2016;10. CD010362.

6 Simpson DM, Gracies JM, Graham HK, et al. Assessment: botulinum neurotoxin for the treatment of spasticity (an evidence-based review): report of the therapeutics and technology assessment subcommittee of the American Academy of Neurology. Neurology 2008;70:1691-8.

7 Esquenazi A, Albanese A, Chancellor MB, et al. Evidence-based review and assessment of botulinum neurotoxin for the treatment of adult spasticity in the upper motor neuron syndrome. Toxicon 2013;67:115-28.
8 Gwartz BL. Intrathecal baclofen for spasticity caused by thrombotic stroke. Am J Phys Med Rehabil 2001;80:383-7.

9 Meythaler JM, Guin-Renfroe S, Brunner RC, et al. Intrathecal baclofen for spastic hypertonia from stroke. Stroke 2001;32:2099-109.

10 Francisco GE, Boake C. Improvement in walking speed in poststroke spastic hemiplegia after intrathecal baclofen therapy: a preliminary study. Arch Phys Med Rehabil 2003;84:1194-9.

11 Rémy-Néris $\mathrm{O}$, Tiffreau V, Bouilland S, et al. Intrathecal baclofen in subjects with spastic hemiplegia: assessment of the antispastic effect during gait. Arch Phys Med Rehabil 2003;84:643-50.

12 Ivanhoe CB, Francisco GE, McGuire JR, et al. Intrathecal baclofen management of poststroke spastic hypertonia: implications for function and quality of life. Arch Phys Med Rehabil 2006;87:1509-15.

13 Lamotte D, Cantalloube S. Efficacy of intrathecal baclofen in the treatment of spasticity in stroke. Ann Readapt Med Phys 2007:50:165-9.

14 Kofler M, Quirbach E, Schauer R, et al. Limitations of intrathecal baclofen for spastic hemiparesis following stroke. Neurorehabil Neural Repair 2009;23:26-31.

15 Schiess MC, Oh IJ, Stimming EF, et al. Prospective 12-month study of intrathecal baclofen therapy for poststroke spastic upper and lower extremity motor control and functional improvement. Neuromodulation 2011;14:38-45.

16 Ashworth B. Preliminary trial of carisoprodol in multiple sclerosis. Practitioner 1964;192:540-2.

17 Granger CV, Cotter AC, Hamilton BB, et al. Functional assessment scales: a study of persons after stroke. Arch Phys Med Rehabil 1993;74:133-8.

18 Shaw L, Rodgers H, Price C, et al. BoTULS: a multicentre randomised controlled trial to evaluate the clinical effectiveness and cost-effectiveness of treating upper limb spasticity due to stroke with botulinum toxin type A. Health Technol Assess 2010;14:1-113. 